\title{
Le baccalauréat et les débuts de la dissertation littéraire (1874-1881)
}

The Baccalauréat and the literary essay at its early stages

Das Abitur und die Anfänge des Literaturaufsatzes (1874-1881)

\section{André Chervel}

\section{(2) OpenEdition}

\section{Journals}

Édition électronique

URL : https://journals.openedition.org/histoire-education/816

DOI : 10.4000/histoire-education.816

ISSN : 2102-5452

Éditeur

ENS Éditions

Édition imprimée

Date de publication : 1 mai 2002

Pagination : 103-139

ISBN : 2-7342-0923

ISSN : 0221-6280

\section{Référence électronique}

André Chervel, «Le baccalauréat et les débuts de la dissertation littéraire (1874-1881) 》, Histoire de l'éducation [En ligne], 94 | 2002, mis en ligne le 12 janvier 2009, consulté le 20 mai 2021. URL : http:// journals.openedition.org/histoire-education/816; DOI : https://doi.org/10.4000/histoire-education.816

Ce document a été généré automatiquement le 20 mai 2021.

(c) Tous droits réservés 


\title{
Le baccalauréat et les débuts de la dissertation littéraire (1874-1881)
}

\author{
The Baccalauréat and the literary essay at its early stages \\ Das Abitur und die Anfänge des Literaturaufsatzes (1874-1881)
}

André Chervel

1 Le baccalauréat ès lettres est profondément modifié en 1881 : la composition latine, qui était l'une des deux épreuves écrites de la première partie, est définitivement supprimée et remplacée par une « composition française sur un sujet de littérature ou d'histoire $»^{1}$. C'est là un tournant décisif dans l'histoire de notre enseignement secondaire, pour au moins deux raisons. La première, c'est qu'il met fin au monopole que le latin exerçait depuis des siècles et qu'il dégage ainsi la voie pour des humanités «modernes ». La seconde, c'est qu'il place au premier rang des objectifs des lycées et collèges un nouvel exercice, la dissertation littéraire, et que cette innovation va bouleverser à la fois l'organisation générale des programmes de français depuis la sixième et les batteries d'exercices pratiqués dans toutes les classes.

On s'attachera ici, à partir de l'histoire du baccalauréat, à éclairer les débuts de la dissertation littéraire en la replaçant dans son contexte historique concret. La petite révolution culturelle de 1881 a été elle-même longuement préparée au cours des années et même des décennies qui la précèdent. La composition latine qui est imposée au baccalauréat jusqu'en 1880 constitue la matrice du nouvel exercice : on étudiera les sujets qui ont été proposés dans cette "faculté », comme on disait à l'époque pour un exercice servant d'épreuve à un examen, et leur traitement par les candidats. La littérature française, qui se trouve brusquement propulsée sur le devant de la scène en 1881, a vu son rôle croître dans l'enseignement secondaire depuis la monarchie de Juillet, et particulièrement depuis 1870 : dans quelle mesure l'évolution des études littéraires menées dans les lycées et collèges a-t-elle contribué à la réforme de 1881 ? Quant à la dissertation littéraire elle-même, qui va commencer à se profiler à l'horizon du baccalauréat en 1881, elle n'est évidemment pas encore au point à cette date : qu'en est-il alors de l'exercice de composition française que les candidats de cette année-là vont devoir rédiger? qu'ont-ils appris à "composer» en français jusque-là? si la 
« dissertation » au sens propre ne fait pas encore partie de leur bagage, quels exercices pratiquent-ils dans les classes et comment vont-ils traiter les sujets qui leur seront proposés? Dernière question. La dissertation figure (depuis respectivement 1810 et 1821) au programme de la licence et de l'agrégation des lettres : comment, et dans quelles conditions, cette épreuve, longtemps réservée aux maîtres et qui n'avait été adoptée que pour les élèves de philosophie, a-t-elle pu « descendre », dans la hiérarchie universitaire, des maîtres vers les élèves, ou dans le cursus des études, des philosophes vers les rhétoriciens? Autant de questions qui seules permettront de prendre la mesure exacte de la transformation que subit en 1881 le baccalauréat ès lettres, des modifications qu'il se prépare encore à subir et, avec lui, tout l'enseignement secondaire classique.

\section{Le baccalauréat et les études classiques : quel est l'enjeu en 1880 ?}

Créé en 1808, le baccalauréat ès lettres est longtemps un examen purement oral, que l'on passe à la fin des études secondaires, c'est-à-dire après la philosophie. En 1840, il reçoit une première épreuve écrite dont la forme est spécifiée officiellement : la version latine. En 1853, Fortoul opère un petit coup d'État universitaire (après le coup d'État du 2 décembre auquel il doit son poste de ministre) en introduisant, contre l'avis de l'ensemble de l'Université, une seconde épreuve écrite, à savoir une composition, qui aura lieu en français ou en latin, "suivant que le sort en décidera ». D'avril 1853 à juillet 1857, la composition française fait donc une première, mais brève, apparition au baccalauréat: dès la mort de Fortoul (1856), les classiques reprennent le dessus et obtiennent de son successeur de ne maintenir que la composition latine, qui règne donc sans partage de 1857 à 1880. L'épreuve est baptisée successivement "discours latin ", «composition latine», «composition en latin $»^{2}$. Mais l'événement important est ailleurs: le règlement de 1874 "scinde" le baccalauréat en deux parties; et ce baccalauréat scindé (c'est l'expression qui prévaut à l'époque) le restera, d'une certaine façon, jusqu'à nos jours, puisque l'épreuve anticipée de français constitue comme une butte témoin de l'ancienne " première partie du baccalauréat ». Le nouveau règlement est appliqué immédiatement : dès le $1^{\mathrm{er}}$ août 1874 , les élèves de la classe de rhétorique (la première) sont autorisés à se joindre à leurs camarades de philosophie, qui passent le baccalauréat suivant l'ancienne formule, pour affronter les mêmes épreuves de composition latine et de version latine. Quant au déroulement quotidien de l'examen, il est, au XIX $X^{e}$ siècle, fort différent du nôtre. À une époque où les professeurs des lycées ne participent pas aux jurys, il impose une tâche écrasante aux facultés des lettres. Le baccalauréat, c'est, en 1880, pour une centaine de professeurs, 6000 journées d'examen, soit une moyenne de dix semaines par an. En réalité, les six facultés les plus chargées font, avec cinquante professeurs, passer les deux tiers des candidats, soit, pour eux, trois mois et demi par an consacrés uniquement au baccalauréat ${ }^{3}$.

4 Le choix de la composition française fait en 1880 est consécutif aux événements politiques de 1879. Les élections sénatoriales du 5 janvier 1879 ont mis un terme à la "république des ducs». Les républicains tiennent désormais toutes les rênes du pouvoir et Jules Ferry prend le 4 février le ministère de l'Instruction publique et des Beaux-Arts. Mais la structure bipolaire de cette administration ne lui permet pas d'opérer immédiatement ses réformes. À côté du ministre qui détient le pouvoir 
exécutif, le Conseil supérieur légifère ${ }^{4}$ en matière de programmes. $Y$ ont longtemps dominé et y dominent encore les évêques et autres notables qui ont fait échouer les réformes de Jules Simon en 1873. La refonte du Conseil supérieur est donc un préalable à toute modification des programmes. La loi du 27 février 1880 en écarte les dignitaires ecclésiastiques et $\mathrm{y}$ accueille les représentants élus des différentes catégories de maitres. Entre temps, l'aile marchante du corps enseignant s'est regroupée dans des sociétés qui fonctionnent comme des groupes de pression. La Société d'étude des questions d'enseignement secondaire, tout en constatant l'état d'impréparation des maîtres en ce qui concerne "l'enseignement du français par le français », décide cependant de se lancer dans l'aventure. Dans sa séance du 5 février 1880, le philosophe Darlu, quoique très hostile au discours latin et favorable à la composition française, ne masque pas les difficultés à venir: «il faudra trouver de nouveaux procédés pour enseigner l'art d'écrire et de penser, car on ne pense et on n'écrit guère de la sixième à la rhétorique qu'en latin ». Le 11 mars suivant, la Société vote une motion appelant à la suppression du discours latin, et le ministère entérine ce bouleversement radical des études classiques en réformant le baccalauréat par le décret et l'arrêté du 19 juin 1880.

5 L'enjeu de la réforme est considérable. Les défenseurs de la tradition continuent, en 1880 comme en 1820, à vanter les mérites des humanités classiques, du latin et de la composition latine dans la formation d'une élite cultivée. Face à eux, les partisans du mouvement plaident que l'enseignement secondaire français s'est trop longtemps attardé dans un mode d'enseignement classique d'un autre âge qui perd peu à peu toutes ses justifications: "Tout a changé dans le monde, écrit un membre de l'Académie des Sciences ${ }^{5}$, et l'Université en est encore au Moyen Âge ». Il faut d'une part faire jouer au français (langue et littérature, auteurs classiques et contemporains) un rôle majeur dans l'éducation et d'autre part accorder une place à des disciplines nouvelles, les langues modernes et les sciences. À tort ou à raison, la défaite de 1871 est imputée par l'opinion à l'arriération des programmes et des méthodes d'enseignement, et ce sont les républicains qui vont se charger de réaliser cette révolution culturelle du secondaire (accompagnant la révolution culturelle du primaire) qui consiste à réorienter l'enseignement secondaire du latin vers le français.

6 Mais on ne peut écarter l'aspect politique du problème. À côté de l'enseignement public masculin qui végète (passant de 79000 à 86000 élèves entre 1876 et 1898) ${ }^{6}$, l'enseignement secondaire catholique connaît une forte progression jusqu'à la fin du siècle (de 47000 à près de 68000 pour la même période). Or il est dans sa grande majorité foncièrement hostile à toutes ces mesures, qui le concernent de près puisqu'il prépare lui aussi ses élèves au baccalauréat. Non seulement il voit disparaître au Conseil supérieur les prélats qui défendaient ses intérêts, non seulement il assiste impuissant au recul du latin, la langue de l'Église, non seulement sa pratique souvent familière de la langue de Cicéron va lui être désormais de peu d'utilité, mais le voilà dans l'obligation de se recycler autour de la littérature française et de la dissertation française, qui n'ont jamais été son fort, et surtout de faire une petite place dans son enseignement à des œuvres pour lesquelles il n'éprouve que de la répulsion, comme les Provinciales ou le Tartuffe, et à des écrivains haïs, comme Voltaire et Jean-Jacques Rousseau ${ }^{7}$, contre lesquels il n'a cessé, depuis la création de l'Université, de fulminer ses imprécations. Pour l'école congréganiste, si la bataille politique est perdue, reste à exercer toutes les pressions qu'elle peut mettre en œuvre pour retarder les évolutions probables et pour favoriser les types d'exercice les plus conformes au modèle périmé, la 
composition historique plutôt que littéraire, le discours ou la narration plutôt que la dissertation ${ }^{8}$.

7 La réforme du baccalauréat de 1880 aura des répercussions sur tout le système des examens universitaires. Le vers latin et la composition latine disparaitront au cours des années suivantes au concours général (respectivement en 1880 et après 1903, date de sa suppression temporaire), au concours d'entrée à l'École normale supérieure (en 1886 et en 1905), à la licence ès lettres (1896 et 1908) et à l'agrégation des lettres (en 1886 et en 1907). À l'ancienne formation rhétorique traditionnelle fondée sur les textes latins, sur leur mémorisation ou sur leur imitation, doit maintenant se substituer une nouvelle formation humaniste : mais celle-ci n'est pas prête. Quelle que soit la bonne volonté des maîtres, ils ne sauraient, dans leur grande masse, passer en quelques années d'un mode d'enseignement à un autre. Certes, au cours du XIx siècle, l'enseignement du français s'est installé dans les classes, où il ne s'était introduit que tardivement au $\mathrm{XvIII}^{\mathrm{e}}$; il a créé ou modernisé des pratiques didactiques, il a familiarisé les élèves avec des textes littéraires, il a mis au point quelques exercices nouveaux (orthographe, narration, lecture des textes), il a officialisé la récitation ${ }^{9}$. Mais, en 1880, les auteurs du XIX siècle sont encore exclus des programmes officiels, on ignore les techniques d'explication des textes français qui prévaudront au $\mathrm{xx}^{\mathrm{e}}$ siècle et les exercices de composition et de rédaction pratiqués en français restent encore plus ou moins soumis aux contraintes de la rhétorique traditionnelle.

Pour les réformateurs, l'objectif général est donc clair, si les moyens de l'atteindre le sont moins. Il s'agit de trouver pour la formation de la jeunesse bourgeoise dans la culture française, dans la littérature française, dans la langue française, l'équivalent de ce qu'on trouvait jusque-là dans la culture antique (langues et littératures grecques et latines). Il ne s'agit pas de supprimer les "humanités », il s'agit de les moderniser. Il s'agit d'adapter, sous la forme d'une nouvelle discipline scolaire, ou d'un nouvel ensemble de disciplines, un pareil objectif aux contraintes propres à l'enseignement. L'enjeu dépasse largement la réforme du baccalauréat. Il repose tout entier sur le bouleversement des études classiques et, pour le baccalauréat, sur la possibilité de trouver une bonne adaptation de l'examen à cette finalité. Depuis plusieurs siècles, on avait présenté la fréquentation des auteurs de l'Antiquité classique et l'exercice du discours latin comme le plus haut sommet de la culture vers lequel on pouvait entraîner les jeunes générations. Et tout à coup, renversement des perspectives : les compositions en latin vont être inutiles et le point culminant de la culture lycéenne doit devenir la composition en français. Le baccalauréat va donc servir de test et de centre d'expérimentation pour la validation de l'exercice.

9 Le problème de la suppression du discours latin a aussi un aspect purement docimologique. L'écrit du baccalauréat comporte de 1857 à 1880 non seulement une composition latine mais une version latine ${ }^{10}$. Or le niveau général de la composition latine, qui n'a jamais été très élevé, s'affaiblit tellement au cours des dix dernières années, que la tendance des correcteurs est soit à la neutraliser, pour confier à la version latine le soin de départager les candidats, soit à ne juger les copies de composition latine qu'à partir du nombre de barbarismes et de solécismes qu'elles comportent : c'est-à-dire qu'on les traite comme des thèmes latins, exercice réservé en principe aux classes de grammaire. Plus précisément, les jurys ont tendance, pour la composition latine, à se réfugier dans la note " passable » qui n'est pas éliminatoire, tandis que l'éventail complet du système de notation est appliqué pour la correction de 
la version latine. Elle devient donc une épreuve inutile puisqu'elle a cessé d'être discriminante, alors que la version latine porte tout le poids de l'examen écrit; et beaucoup de professeurs, même s'ils sont attachés à l'exercice, en réclament pour cette raison la suppression.

\section{Les exercices de composition dans l'enseignement secondaire avant 1880}

10 Il convient d'abord de revenir sur une idée reçue largement répandue, selon laquelle en matière d'enseignement $\mathrm{du}$ français, tout commence en 1880. L'étude de l'enseignement traditionnel des humanités classiques pratiqué au XIX ${ }^{e}$ siècle oblige à faire une constatation importante, qui va à l'encontre de l'appréciation qu'on porte généralement sur lui : écrire en français, sur l'ordre du professeur et dans le cadre d'exercices consacrés par la tradition, c'est déjà, avant 1880, l'activité principale des classes. Certes, l'élève « étudie » plus le latin que le français, c'est-à-dire qu'il connaît sans doute un peu mieux les verbes déponents que l'accord des participes passés et qu'il a passé plus de temps sur l'Enéide ou sur Tite-Live que sur Corneille ou Montesquieu. Mais, au total, il consacre au cours de sa scolarité beaucoup plus de temps à écrire en français qu'à écrire en latin. Les exercices de français qu'il pratique quotidiennement peuvent être classés en plusieurs niveaux en fonction de leur statut scolaire ${ }^{11}$. La réglementation officielle ne mentionne jamais qu'une partie d'entre eux, ceux qui donnent matière à des prix. Mais ne nous laissons pas abuser par les programmes officiels et les directives ministérielles. Dans l'activité quotidienne de la classe, l'autonomie du maître par rapport aux programmes et aux directives est considérable, ce qui peut s'exprimer d'une autre manière : les règlements officiels (décrets, arrêtés, circulaires) restent totalement silencieux sur toute la partie non problématique de la pédagogie classique. Pour la hiérarchie, pour les chefs d'établissement, pour l'inspection générale, tout cela va sans dire. Les usages invétérés de la classe, les habitudes didactiques héritées du passé, les humbles pratiques de lecture, de notation, de formulation écrite, de correction, de recopiage, de gloses diverses qui sont liées à l'explication des textes latins (et grecs) ou au compte rendu des devoirs par le maître, tout le quotidien de la classe et de l'étude risque d'échapper au regard qui resterait obstinément fixé sur les instructions officielles. À négliger ces aspects apparemment mineurs, on court le risque de méconnaître le fond même de la pédagogie traditionnelle et surtout de mal interpréter les bouleversements qu'elle a connus autour de 1880 .

11 Comment, avant la réforme de 1880, est organisé à l'intérieur des classes l'enseignement $d u$ français et plus particulièrement l'enseignement de la rédaction en français, de l'aptitude à s'exprimer par écrit en français, à composer en français? Quelle est la hiérarchie des exercices en usage, du plus simple au plus relevé ? C'est le lieu de rappeler que toute discipline fonctionne avec un éventail d'exercices hiérarchisés, les uns visant à une "gymnastique ", à une " mise en jambe ", à faire des gammes, les autres étant au contraire des objectifs nommément désignés de cette pédagogie, des exercices de prestige, ceux qui trouvent leur place dans les examens et concours, ceux sur lesquels les élèves sont mis en compétition au moment des compositions ou des examens, ceux qui donnent matière à des " prix », dont la liste est 
fixée par le ministère. Procédons du plus élémentaire au plus élaboré, du plus humble au plus noble.

Un exemple, qui vaut pour l'ensemble de la scolarité. Le pain quotidien de l'élève, c'est «l'explication» latine. L'exercice se fait en deux temps. Avant la classe, chez lui ou en étude, l'élève, muni de son dictionnaire, écrit sur son cahier une traduction mot à mot du passage imposé par le professeur : c'est la «préparation de l'explication $»^{12}$. Pendant la classe, le professeur donne une traduction "en bon français ", comme on dit déjà à l'époque. Après la classe, l'élève est tenu de rédiger le même texte sur ce modèle, en français correct, toujours sur son cahier: c'est ce qu'on appelle le "français de l'explication », ou le " rapporté ». Humble exercice, qui prépare à la version latine, ellemême longtemps considérée essentiellement comme un exercice de français; et c'est à ce titre que Victor Cousin en avait fait la première épreuve écrite du baccalauréat.

Dans les grandes classes, l'exercice principal, celui qui occupe la plus grande partie du temps de travail à la maison ou à l'étude, c'est la rédaction du cours. Chaque fois que l'enseignement donne lieu à un cours, les élèves sont tenus d'en faire la "rédaction ». Elle est faite uniquement sur cahier : ce n'est donc pas un « devoir». Il y a rédaction en rhétorique, en histoire littéraire, en histoire de la langue française ${ }^{13}$, en géographie, etc. Il y a surtout rédaction en histoire. Après le cours d'histoire, l'élève, rentré chez lui, ou à l'étude, doit reproduire par écrit, de mémoire, en s'aidant de ses notes, du manuel ou du mémento, le cours du professeur. Le lendemain, le maître vérifie le travail en faisant lire quelques rédactions, ou en relevant des cahiers. Naturellement, la rédaction du cours est un exercice mineur, pour lequel aucun prix n'a jamais été décerné. Ses inconvénients : on écrit sa rédaction trop rapidement, sans soigner son style, sans s'attacher à l'exactitude de la pensée, sans éviter les banalités. Mais elle est appréciée parce qu'elle accoutume l'élève non seulement à écrire des phrases françaises, mais à les enchaîner d'une façon cohérente dans des textes que beaucoup de professeurs veulent très longs.

Venons-en aux exercices dits, à l'époque, « de composition ». Eux aussi ont pour origine un texte (écrit ou oral), plus ou moins long. Mais, à la différence du précédent, ils se tiennent un peu plus loin du texte de départ, ou du cours du professeur : ils laissent un peu de place à l'« invention ». Le plus important, et de loin, est un exercice qui porte le nom d'« analyse » ou d'« analyse littéraire » et qui est aujourd'hui totalement inconnu, du moins sous ce nom, de nos pratiques pédagogiques. Rien à voir avec les analyses faites en grammaire. C'est un exercice scolaire bien défini, quoiqu'il comporte des variantes, ou plus exactement, une forme simple et une forme plus riche. Son principe consiste à «rendre compte » d'un texte, qu'il s'agisse d'une œuvre complète, ou d'un extrait. Dans sa forme simple, c'est un résumé ${ }^{14}$ mettant l'accent sur l'idée principale et ses relations logiques avec les idées secondaires. Les formes plus riches y ajoutent des citations, des informations sur l'auteur, des réflexions sur le texte, des comparaisons avec d'autres textes, des appréciations littéraires. Dans la réglementation officielle, il est longtemps considéré, pour l'enseignement classique, comme un exercice « implicite », qui ne mérite pas de mention particulière parce qu'il n'est pas couronné par un prix de fin d'année, mais qui est recommandé dans plus d'un texte officiel. En 1852, il est enfin mis officiellement au programme de la rhétorique et de la seconde. Il gagne même la troisième à partir de $1874^{15}$. C'est-à-dire que l'élève qui présente la première partie du baccalauréat en 1881 s'est exercé pendant trois ans à résumer des textes, des scènes, des poèmes, des tragédies. À la fin de 1878, une enquête 
ministérielle, qui sera exploitée par les services de Jules Ferry, montre que l'analyse littéraire est devenue l'exercice le plus important des classes de rhétorique et de seconde.

Traditionnellement, ce n'est pas avant la classe de rhétorique que les élèves rédigent des compositions de leur cru: nul ne doit prendre la plume pour produire quelque chose de personnel s'il n'a appris les règles de la composition, de la narration, du discours, de la poésie (il n'est question que de vers latins, le vers français étant proscrit). Cette conception intellectualiste est peu à peu remise en cause dans de nombreux établissements au cours du XIx ${ }^{\mathrm{e}}$ siècle et la narration (latine, puis française) s'impose d'abord en classe de seconde ; puis des exercices de composition relativement libres se répandent dans les plus petites classes au cours de la seconde moitié du siècle. Mais, jusqu'en 1874, le règlement ne prévoit pas d'exercices de composition avant la classe de troisième. Quant aux petits établissements de province, ils se sont depuis longtemps affranchis de ces servitudes et font volontiers composer dans toutes les classes.

Le discours, pratiqué en rhétorique, est l'exercice emblématique de l'enseignement classique. La définition du discours est très large. Il y a discours «toutes les fois qu'un ou plusieurs personnages s'adressent directement, soit de vive voix, soit par écrit à un ou plusieurs auditeurs ou correspondants ${ }^{16}$. La lettre en est donc une variante, un peu moins oratoire. Mais en dépit de son caractère prestigieux, le discours n'est, vu l'ensemble des qualités et le niveau de culture qu'il requiert, pratiqué d'une façon efficace que dans les grands établissements qui préparent au concours général et au concours de l'École normale, c'est-à-dire surtout les lycées parisiens. En province, l'enseignement secondaire ne sera vraiment concerné qu'au moment où les recteurs commenceront à organiser des concours académiques entre établissements, soit à partir de 1856. Mais il ne figure pas dans les épreuves des concours d'entrée des grandes écoles militaires: Polytechnique, Saint-Cyr, Navale se contentent d'une narration, car beaucoup de candidats n'ont pas fait la classe de rhétorique, ou de petites dissertations sur des thèmes moraux, patriotiques ou guerriers, et largement soutenues par une «matière » abondant ${ }^{17}$. Pas de « discours » non plus aux examens et concours de recrutement du corps enseignant, la licence et l'agrégation, où les candidats sont jugés sur des dissertations françaises, qui mettent en jeu d'autres qualités.

\section{La littérature française dans l'enseignement avant 1880}

17 Dans l'enseignement classique hérité de l'Ancien Régime, la science de la littérature, baptisée tantôt "rhétorique », tantôt « littérature ", fait l'objet d'un cours magistral qui vise à enseigner les bases théoriques de la composition. Le professeur de la classe de rhétorique administre à ses élèves un " cours de rhétorique » qui est généralement pris sous sa dictée et qui est supposé favoriser l'aptitude à rédiger des amplifications dans un style noble et abondant. Les bibliothèques de province conservent aujourd'hui encore quelques centaines de cahiers d'élèves ${ }^{18}$ reproduisant le cours de leur maître, en latin jusque vers 1760 , en français ensuite. Il s'agit d'un enseignement dogmatique portant sur les différents styles (il y en a trois, le simple, le tempéré et le sublime), sur les genres en prose et en vers, sur l'invention, la disposition et l'élocution, sur les lieux 
communs, les figures, etc. Dès l'origine, le programme du baccalauréat comporte une interrogation de rhétorique. En 1840, Victor Cousin publie pour la première fois un programme de cinquante questions de «littérature » qui seront posées aux candidats, par tirage au sort: on y retrouve l'essentiel de la rhétorique des collèges, mais également, et c'est une petite nouveauté, quinze questions d' " histoire littéraire », qui ne portent d'ailleurs que sur des généralités et qui sont équitablement réparties entre les trois littératures classiques.

18 C'est encore Fortoul qui met un terme à l'interrogation orale de rhétorique du baccalauréat ${ }^{19}$. Le candidat est seulement tenu désormais de "répondre à toutes les questions littéraires qui [lui] seront faites » à propos des textes que l'examinateur lui demande d'expliquer ${ }^{20}$. Le cours magistral de rhétorique, qui était pratiqué depuis au moins le XvII ${ }^{\mathrm{e}}$ siècle, disparaît donc vers le milieu du $\mathrm{XIX}^{\mathrm{e}}$, au profit d'un enseignement «littéraire » organisé autour des différents "genres ». C'est ce que confirme Petit de Julleville quand il constate en 1868 que «l'enseignement de la rhétorique est maintenant omis dans la classe qui porte encore ce nom ${ }^{21}$.

Quant à l'enseignement de l'histoire de la littérature française, il reste matière d'enseignement supérieur jusqu'à la guerre de 1870. C'est alors que se produit dans les établissements secondaires un phénomène qui n'a guère été observé jusqu'ici. Un certain nombre de textes officiels publiés entre 1872 et 1875 mettent en évidence une évolution profonde et rapide de l'état d'esprit et de l'enseignement des professeurs de lettres des grandes classes, de la rhétorique à la troisième. Une tendance croissante se manifeste dans les classes au cours des années 1870 en faveur de l'enseignement de l'histoire littéraire, mais également en direction d'une littérature plus récente que celle des auteurs du programme et en particulier vers les écrivains du XIX $x^{e}$ siècle, dont aucun n'a encore trouvé sa place dans les listes ministérielles. Voici les textes officiels de la période, ici mentionnés comme révélateurs de cette pression:

- 27 septembre 1872. - Circulaire (ministérielle) aux proviseurs sur l'enseignement secondaire ${ }^{22}$. Jules Simon s'y déclare partisan de la composition française généralisée et de l'histoire littéraire : « Je désire donc que, dans toutes les classes, une part, judicieusement mesurée, soit faite aux exercices français, depuis les classes les plus élémentaires [...]. À l'introduction d'exercices et de compositions en français dans toutes les classes se rattachera utilement l'étude sommaire de la langue et de la littérature françaises, dans leur origine et leurs développements. Nos élèves sont trop étrangers à l'histoire littéraire ».

- 14 décembre 1872. - Nouvelle circulaire de Jules Simon (aux recteurs, sur l'enseignement de la langue et de la littérature françaises), qui, face à la véhémence des réactions ${ }^{23}$ suscitées par la précédente, fait officiellement marche arrière : « Je n'ai jamais songé à introduire dans les lycées un cours proprement dit d'histoire littéraire [...]. J'ai toujours pensé que l'histoire littéraire devait s'apprendre dans les classes d'humanités par la lecture et par l'explication des auteurs sans en faire l'objet d'un enseignement spécial. C'est donc à tort que MM. les professeurs ont dressé le plan d'un cours d'histoire de la langue et de la littérature françaises qui serait réparti entre les classes de troisième, de seconde et de rhétorique ».

- 23 juillet 1874. - Le nouvel Arrêté sur le plan d'études des lycées avalise l'évolution en cours et met au programme de la classe de rhétorique « l'étude de la langue et de la littérature françaises ». Une fois de plus, on constate qu'une des fonctions de la réglementation officielle consiste moins à promouvoir des innovations issues des réflexions des cercles dirigeants qu'à entériner l'évolution naturelle du système pédagogique et que bien des 
décisions officielles trouvent leur origine dans les salles de classe. Mais l'administration va simultanément s'efforcer de freiner le mouvement.

- 5 avril 1875. - Circulaire du vice-recteur de l'académie de Paris (Adolphe Mourier) aux inspecteurs de l'académie, relative à l'envoi d'un programme pour l'enseignement du français dans les lycées et

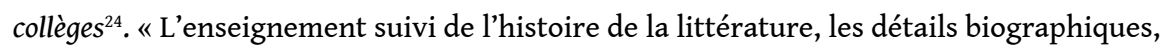
critiques, philologiques, ont pris une place exagérée, souvent dès la troisième, dans certaines classes de nos lycées [...]. Il appartient également au professeur, en seconde et en rhétorique, de désigner pour l'histoire littéraire et pour celle de la langue, les livres, traités et recueils que les élèves pourront consulter avec fruit, et dont on contrôlera la lecture ».

- 8 novembre 1875. - Circulaire (ministérielle) relative à l'enseignement littéraire (Henri Wallon). « Divers renseignements que j'ai recueillis me font craindre quelques écarts, contre lesquels il est bon de prémunir les maîtres qui seraient tentés de s'y laisser entraîner [...]. Je ne saurais blâmer en elles-mêmes des lectures discrètes, propres à initier les élèves aux beautés les plus pures de notre littérature du dix-neuvième siècle. Mais on comprend que beaucoup de réserve est nécessaire ; il ne faut pas que l'attrait plus vif des œuvres contemporaines détourne les jeunes gens de l'étude des auteurs classiques, qui sont la base de tout solide enseignement littéraire ».

En trois ans, les textes officiels sur l'orientation de l'enseignement littéraire se sont multipliés. Jamais l'Université n'avait été confrontée à une contestation aussi générale de ses programmes ${ }^{25}$. Le traumatisme consécutif à la défaite de 1871 et à l'amputation du territoire national a visiblement provoqué dans l'ensemble du corps enseignant un sursaut, une réaction patriotique qui, dans les lycées et collèges, se concentre autour de la littérature française (et de la langue française). Dès la fin de la guerre, un peu comme une lame de fond, il semble que partout les professeurs et les élèves accordent soudain un intérêt particulier à la littérature nationale, à son histoire, aux œuvres récentes. Les témoignages d'archives ${ }^{26}$ font état de nombreuses décisions prises par les professeurs dans des réunions organisées en 1872 par les chefs d'établissements et communiquées au ministère. Au lycée de Bourges, par exemple, il est décidé (18 novembre 1872) que les discours français de rhétorique seront désormais «des discours-dissertations traitant uniquement de sujets littéraires et permettant aux élèves de faire une riche moisson d'idées littéraires et morales ". On n'expliquerait pas le succès si rapide de la dissertation littéraire au lycée si l'on ne tenait compte des mouvements profonds de l'opinion, et du corps enseignant en particulier. Le ministère, qui, après Jules Simon, s'attache à endiguer le mouvement, se voit cependant dans l'obligation de lui accorder une satisfaction partielle et de légitimer les initiatives déjà prises par des dizaines de professeurs qui ont transformé en cours d'histoire littéraire les généralités sur la littérature dont ils se contentaient jusque-là. Faute d'une vision à plus long terme, dans une période il est vrai fort incertaine, il va ainsi favoriser la mise en place d'un exercice particulièrement insolite.

\section{La composition latine de littérature française}

21 Au baccalauréat d'août 1874 , les élèves de rhétorique sont invités pour la première fois à passer deux épreuves écrites ${ }^{27}$, la version et la composition latines, qui étaient réservées jusque-là à leurs camarades de philosophie. Ce sont les doyens des facultés des lettres qui fixent les sujets dans toutes les disciplines, qu'il s'agisse de version, de discours latin ou de dissertation philosophique ${ }^{28}$; et ce sont les professeurs de la faculté 
qui corrigent les copies. À chaque session, les jurys sont appelés à se déplacer dans d'autres "centres de baccalauréat" dont le nombre, une petite vingtaine, est fixé chaque année par arrêté ministériel : autant de sujets nouveaux à proposer. Et comme les candidats sont répartis par séries de vingt qui passent les unes après les autres, comme il y a trois sessions dans l'année, c'est chaque fois un nombre important de sujets qu'il faut établir.

Si les copies du baccalauréat des années 1850-1900 ont quasiment toutes disparu ${ }^{29}$, on connaît cependant une partie des sujets qui ont été proposés, qu'ils aient été publiés dans les périodiques proches de l'Université ${ }^{30}$ ou dans des recueils spécifiques. Entre avril 1853 et novembre 1880, dernière session comportant une " composition en latin ", 1281 sujets de composition latine ont pu être relevés. Dans ce nombre, les 861 sujets parisiens sont largement majoritaires. À l'inverse, certaines des quinze facultés de province sont à peine, ou pas du tout, représentées dans ce tableau : on n'a qu'un seul sujet pour Besançon, Clermont et Montpellier et aucun pour Strasbourg ${ }^{31}$, Nancy et Poitiers. On peut estimer que le nombre des sujets réellement donnés est au moins trois ou quatre fois supérieur.

Ce n'est pas ici le lieu de présenter l'évolution de la composition latine au baccalauréat pendant ses vingt-huit années d'exercice, sous le Second Empire et dans les débuts de la Troisième République. Deux mots cependant à ce propos. On a évoqué plus haut la régression de 1857 qui, après la mort de Fortoul, avait supprimé l'épreuve de composition française. C'est une régression analogue qu'on observe à partir de la même année dans les sujets de la composition latine. Elle est désormais baptisée "discours latin ", ce qui la ramène aux artifices et aux pompes de la rhétorique. De fait, à partir de cette date, plusieurs centaines des sujets relevés (au moins 350 ) sont des discours du type le plus conventionnel et donc le plus artificiel, tel ce sujet donné à Toulouse en 1869 et dont le libellé était exceptionnellement rédigé en français : « Un chef barbare fait prisonnier sous Néron annonce à ses compagnons de captivité que le lendemain, aux jeux du cirque, ils doivent combattre entre eux comme des gladiateurs ou contre les bêtes féroces ${ }^{32}$. Et, ce qui est notable, c'est que lorsque Duruy remplace en 1864, dans le libellé de l'épreuve, "discours latin » par " composition latine », qui rendait aux facultés la possibilité de diversifier leurs sujets et de sortir des sentiers battus, ce sont encore des discours latins qui dominent, pendant plusieurs années : visiblement, les professeurs des facultés ne tiennent pas à modifier leurs habitudes.

Pourtant, dès 1869, quelques signes prémonitoires annoncent les bouleversements à venir. Deux sujets donnés à Douai en novembre, un portrait et un discours, renvoient à la tragédie française classique : "In tragoedia quae dicitur Polyeuctus, Polyeucti personam effingetis $»^{33}$ et " Mardochaeus ad reginam Esther, quum illa regem Assuerum supplex pro Judaeis adire, metu mortis, dubitaret $~_{34}$. En avril 1873, la Sorbonne demande de comparer La Fontaine, Phèdre et Esope (Fontanium cum Phaedro et Aesopo conferetis). Le 26 juillet de la même année, soit un an avant la réforme du baccalauréat, les candidats ont la surprise d'entendre l'examinateur leur dicter un sujet de pure littérature française: "Athaliae et Agrippinae mores, ingenium et personam apud Racinium comparentur $»^{35}$. Jamais la chose ne s'était encore produite à Paris ${ }^{36}$. Jamais la littérature française n'avait été jusqu'alors convoquée au baccalauréat, dans deux de ses plus grandes œuvres, pour servir de matière à une composition littéraire, qui reflète incontestablement une évolution en cours dans les lycées et collèges. Certes, dans les années Fortoul, à l'époque où la composition était française ou latine "suivant que le sort en décidera ", 
on leur avait parfois demandé de raconter une fable en latin, le Chêne et le Roseau, "Narrabis fabulam de quercu et arundine " (1853), ou le Rat de ville et le Rat des champs, «Narrabitur fabula de mure urbano et de mure rustico » (1855). Mais, outre que ces mêmes fables n'étaient pas forcément de La Fontaine, puisqu'elles avaient pu être aussi bien étudiées dans Esope ou dans Horace ${ }^{37}$, deux auteurs du programme, outre que le latin avait pu n'être qu'un accident du tirage au sort, une narration n'est jamais qu'une narration, même sous la plume d'élèves qui sortent de la classe de philosophie ${ }^{38}$. Cette fois-ci, la littérature française était bien seule en cause. L'épreuve proposée ce 26 juillet 1873 était un exercice bien connu à l'époque, qu'on appelait le " parallèle », entre deux personnages, entre deux œuvres, entre deux auteurs, ou entre deux grands hommes. Il avait plusieurs objets : vérifier si le candidat connaissait les tragédies en question, s'il était capable de présenter des remarques, des observations, de mener des raisonnements élémentaires... et, en l'occurrence, s'il pouvait s'exprimer en latin. Le véritable virage date d'août 1874 : la littérature française commence alors à s'installer solidement à l'intérieur de la composition latine du baccalauréat. Le nouveau règlement, en appelant les élèves de rhétorique à passer cette épreuve, a contribué à ce résultat inattendu, car c'est bien dans la classe de rhétorique et non en philosophie qu'on se prépare à la composition latine et qu'on étudie la littérature française.

Les types de sujets proposés sont variés. Le parallèle est toujours aussi recherché, d'une pièce à l'autre, ou dans la même pièce: "Mathan in Athalia et Narcissus in Britannico comparentur»; "Burrhi et Narcissi personae apud Racinium opponentur»"39. On compare ainsi Chimène et Pauline, Polyeucte et Sévère, Horace et Curiace, Aman et Mardochée, l'Art poétique d'Horace et celui de Boileau. Il s'agit également de vérifier les connaissances acquises dans le cours d'histoire littéraire : que savent-ils de la doctrine de Boileau en matière de poésie épique (Quae sint apud Bolaeum de epico poemate praecepta memorabis) ou de tragédie (Quaenam sint apud Bolaeum de tragoedia regulae), que saventils sur les rôles de pères chez Corneille (Quae sint apud Cornelium paternae indolis partes, exemplis additis memorandum); peuvent-ils traiter les grandes questions de l'histoire littéraire (Des poètes épiques. - Quinam praecipui apud veteres et recentiores in epico carmine poetae floruerint $)^{40}$ ? Les fables de La Fontaine sont abordées sous l'angle de la morale (Utilité des fables. - Quaenam sit ad informandos mores fabularum utilitas), des personnages favoris du fabuliste (Quae sint praecipuae in Fontanii fabulis personae memorabis), ou par le biais de l'analyse (Les animaux malades de la peste.- Aegrotantium peste animalium fabulam narrabis).

Même en matière de littérature française, le discours latin n'a pas encore perdu tous ses droits: Boileau offre de renoncer à ses honoraires et demande à Louis XIV de rétablir la pension de Corneille vieillissant et sans ressources (Verba Bolaei ad regem rogantis ut annua sibi adimantur, Cornelio autem, maximo poetae seni atque inopi, restituantur), Esther défendant la cause des Juifs devant Assuérus (Esther coram Assuero rege Judaïcae gentis causam agit), Polyeucte résistant aux supplications de son épouse (Polyeuctus Paulinae precibus resistit) ou Burrhus exhortant Néron à retrouver le chemin du devoir (Burrhus Neronem admonet ne priore virtute sua desciscat).

Certes, ce ne sont pas tous les auteurs du programme de français qui pénètrent dans ces dissertations latines: Pascal, La Bruyère, Buffon, Le Siècle de Louis XIV ne semblent pas concernés par cette nouvelle mode; Molière y est rare. Nombreux sont, au contraire, les sujets sur La Fontaine, sur Fénelon, surtout sur la tragédie classique, 
systématiquement exploitée d'Esther à Britannicus, ou du Cid à Polyeucte, jusque dans les œuvres étudiées dans les petites classes.

Au total, d'août 1874 à fin 1880 , ce sont 27 sujets de littérature française à traiter en latin qui ont été retrouvés, sur un total de 291 sujets donnés pendant la même période ${ }^{41}$, soit près de $10 \%$. Mais la considération de la seule littérature française ne rend pas compte de l'importance de l'évolution en cours. Beaucoup plus nombreux encore sont, à la même époque, les sujets de composition latine portant sur les littératures antiques. Il peut s'agir de discours (Discours de Vercingétorix à Alésia, fondé sur les Commentaires de César), de narrations (Récit du gouverneur d'Oreste. - Orestis paedagogus, nuntii personam agens, Clytemnestrae ficta narratione exponit quomodo Orestes in ludis pythicis perierit ${ }^{42}$; Ruine de Troie. Narretur Trojae excidium ; Enée après la prise de Troie. Aeneae errores post Trojae excidium narrentur) ${ }^{43}$, de parallèles (Demosthenis et Ciceronis vita et mors comparentur; Aeneas et Turnus apud Virgilium comparentur ${ }^{44}$; Parallèle d'Achille et d'Enée. - Achilles et Aeneas comparentur), d'analyses d'oeuvres du programme : l'Aululaire de Plaute (Aululariae Plautinae fabulae brevis expositio), l'Enéide (Aeneidos libri et personae breviter memorentur), la tragédie de Philoctète (Philoctetae tragoediae Sophocleae ratio, personae, exitus $)^{45}$.

30 L'abondance des sujets littéraires (plus de la moitié sans doute, du moins à Paris) dans les compositions latines du baccalauréat est significative d'une évolution qui ne peut que condamner à terme (et le terme est proche) le genre même de la composition latine. Quand le discours latin et la narration latine régnaient dans les classes et dans les salles d'examen, la justification de l'exercice, constamment répétée, était la théorie de l'imitation. L'élève imitait les harangues de Tite-Live, réunies dans le Conciones, ce Tite-Live dont Taine avait montré ${ }^{46}$ qu'il était beaucoup plus un orateur qu'un historien, ou les récits des historiens latins réunis dans le Narrationes (c'était encore du Tite-Live, pour l'essentiel). Avec les compositions latines sur des sujets de littérature française, l'élève n'imite plus rien, ni les auteurs latins, ni les manuels mis à sa disposition, ni même ses maîtres. Car, pendant ces quelques années, les professeurs de rhétorique préparent leurs élèves à une épreuve d'un type totalement nouveau, la composition latine de littérature française, à laquelle ils n'ont pas été formés euxmêmes et qu'ils n'ont jamais eu à affronter ni pendant leur scolarité, ni au baccalauréat de leur jeunesse, ni pour la licence, ni pour le recrutement au professorat.

31 Et pourtant la chose aurait bien pu se produire. Le concours d'agrégation comme la licence comportaient au XIX ${ }^{e}$ siècle une composition latine. Mieux encore : le règlement de l'agrégation des «classes supérieures des lettres» du 27 décembre 1828 fixait comme thème de cette épreuve une "dissertation latine sur un sujet de littérature ancienne ou moderne ». Les candidats à l'agrégation eurent-ils alors à traiter en latin des sujets de littérature française ? Il n'en est évidemment rien : les sept sujets donnés dans le cadre de ce règlement ${ }^{47}$ traitent de littérature générale ou de littérature antique, par exemple, en 1833, «Praecipuas causas afferre, cur Romae, post Caesaris Augusti tempora, scribendi genus corruptum fuerit $»^{48}$. Le jury du concours se garde bien du ridicule de faire composer en latin sur la littérature française et, sans tenir compte du règlement de 1828, qui fixait pour la composition en français une "dissertation française sur un sujet de philosophie ", il donne à traiter en français, en 1836, un sujet qui n'a rien de philosophique: «Entretien entre Condé, Balzac et Scudéry, sur les premières tragédies de Corneille $»^{49}$. Il ne reste plus au ministère qu'à changer son fusil d'épaule et à modifier le règlement en conséquence: dès 1837, c'est dans la 
« composition en prose française» que les candidats devront traiter la question de littérature ancienne ou moderne ${ }^{50}$. La composition en prose latine porte désormais sur la « philosophie morale ».

32 La licence ès lettres, qui était l'une des voies d'accès à l'enseignement secondaire, comportait elle aussi une composition «en prose latine ». Pour la période 1815-1880, 467 sujets ont été retrouvés, toutes les facultés ayant apporté leur écot. Parmi eux, une dizaine mentionnent la littérature française, mais aucun, sauf le dernier en date, n'est comparable aux sujets du baccalauréat 1874-1880. Il y a d'abord les sujets très généraux du type de ceux qui prévalaient jusque vers 1830 , comme "Tantum gloriae comparavit Gallia scriptis quantum victoriis " ${ }^{51}$, donné à Caen en 1821 ; puis des sujets philosophiques ou moraux, portant sur des œuvres qui ont été depuis lors annexées au corpus littéraire de l'enseignement secondaire, mais qui ne l'étaient pas à l'époque, comme « Apprécier la lettre de Jean-Jacques Rousseau sur les spectacles » (Toulouse, 1855); enfin des sujets mettant en parallèle littérature antique et littérature française comme "Virgilius et Racinius inter se conferuntur $"{ }^{52}$ (Dijon, 1855). Un seul sujet de composition latine de licence porte sur une question précise de littérature française : «Racinius ostendatur in Andromache sua inventor exstitisse novi generis quod a summi judicii viro dictum est tragoedia graeco-gallica $»^{53}$. Il est donné à Besançon en avril 1878 alors que depuis plusieurs années déjà la littérature française figure dans l'épreuve latine du baccalauréat. C'est la licence qui se rapproche du baccalauréat, et non l'inverse.

La composition latine de littérature française est certes une création originale de l'Université française, mais il serait difficile de pousser plus loin l'éloge de ce nouvel exercice aussi stérile que grotesque. Le philosophe Paul Janet, solide partisan des réformes de 1872, formule contre elle des critiques dont elle ne pouvait se relever : «si [les élèves] sont incapables de composer en français, on ne voit pas comment une difficulté de plus les rendrait plus capables de composer dans une langue étrangère. Tout ce qu'on peut dire, c'est que la platitude se voit moins en latin qu'en français et qu'elle est peut-être moins choquante parce qu'elle est tempérée par l'incorrection. Les barbarismes et les solécismes qui sautent aux yeux permettent de ne pas trop faire attention à la sottise du fond $\aleph^{54}$. Partialité excessive d'un correcteur excédé qui n'a gardé le souvenir que des mauvaises copies? Véhémence d'un moderne, proche de Jules Simon et soucieux de faire mouche avec des arguments chocs? Absolument pas : les défenseurs de la composition latine à l'époque ne s'expriment pas autrement. Tous les griefs des adversaires de la composition latine sont pour eux des arguments favorables. Voici par exemple le manuel de composition littéraire du baccalauréat ${ }^{55} \mathrm{du}$ professeur Charles Aubertin, qu'on va retrouver un peu plus loin dans la chaire de littérature française à la faculté des lettres de Dijon. Le manuel s'adapte aux tribulations de l'épreuve; et la réédition de 1878 se borne, et pour cause, aux compositions latines. Aubertin justifie cette épreuve pourtant bien décriée avec la plus parfaite ingénuité : «Que faut-il pour réussir au baccalauréat [en composition latine] ? Respecter la syntaxe et éviter les gallicismes. Si le développement est incomplet, si le sujet est détourné de son vrai sens, s'il y a quelque embarras dans l'exposition, ces fautes obtiennent grâce, pourvu que l'auteur témoigne, par un souci avoué de la grammaire et du bon goût, par des réminiscences bien placées, que la langue latine ne lui est pas étrangère. Prêts à récompenser les moindres mérites, les correcteurs lui tiennent compte de tous ses efforts. En français, au contraire, le vague des idées, le désordre et la stérilité de l'esprit sont mis à nu et se trahissent avec un éclat fâcheux; rien ne dissimule, rien n'atténue les fautes; on ne vous sait aucun gré d'une sorte de 
diffusion banale qui ne marque ni esprit ni travail; la critique des juges s'exerce sévèrement sur le fond des choses, car vous n'avez pas pour excuse la gêne de l'expression ". Au cours des années 1870, on sent monter la contradiction entre une nouvelle discipline qui s'impose dans l'enseignement secondaire, la connaissance de la littérature et de l'histoire littéraire, et d'autre part une forme périmée, une contrainte de plus en plus insupportable, un cadre totalement inadapté, à savoir l'utilisation de la langue latine pour l'expression de cette discipline nouvelle.

Dans ces conditions, la composition latine ne peut que se dégrader en thème latin. Quand la lecture, la traduction et l'imitation des discours latins permettaient d'accéder à une culture humaniste, la rédaction par l'élève de son discours latin était un exercice fructueux, aussi élitiste et suranné fût-il, puisqu'il pouvait y exploiter les éléments de la culture antique qu'il avait acquise pendant ses années de collège. Quand il s'agit de traiter en latin des questions qui, en classe ou dans les manuels de littérature, n'ont jamais été abordées qu'en français, l'élève rédige mentalement en français et s'efforce de traduire en latin les parties les plus banales de sa rédaction, quitte à renoncer à s'exprimer lorsqu'il trouve une pensée plus délicate ou plus audacieuse dont la traduction lui pose problème. Aux différentes causes du triomphe de la composition française en 1881, il convient donc d'en ajouter une nouvelle, de nature purement disciplinaire : c'est la littérature française elle-même qui, de l'intérieur, fait exploser la forme « latine » donnée aux élèves pour s'exprimer.

\section{Les professeurs de littérature française des facultés des lettres en 1881}

Quand, en juillet 1881, la composition française se substitue à la composition latine comme épreuve écrite de la première partie de l'examen, ce sont, dans toutes les facultés des lettres de France, les mêmes doyens qui sont en place et les mêmes professeurs de littérature française que l'année précédente. À la différence des sujets de composition latine dont le contenu n'était pas spécifié par le règlement, les voilà désormais contraints de limiter à la littérature ou à l'histoire le libellé des épreuves.

Le très petit nombre des sujets de composition latine retrouvés pour les deux dernières années de cette épreuve (1879 et 1880) ne nous permet pas de dessiner avec précision la courbe du changement au cours de la période de transition. On dispose cependant des vingt-deux sujets ${ }^{56}$ donnés à Paris lors de la troisième et dernière session de 1880 . Dixsept d'entre eux portent d'une façon ou d'une autre sur les trois littératures (dont deux pour la littérature française) et cinq seulement ${ }^{57}$ sont des discours du type traditionnel. La transition vers la littérature semble donc s'être opérée très graduellement. Sur ce plan au moins, le passage du latin au français ne marque pas un bouleversement complet.

Si les sujets sont fixés par les doyens, si l'ensemble des membres de chaque faculté sont associés à la correction des copies, il ne fait en revanche aucun doute que les professeurs de littérature française ne sont désormais particulièrement sollicités. On va voir en effet que, dès la première année, près de la moitié des sujets seront pris dans leur domaine. Qui sont ces professeurs de littérature française des quinze facultés ${ }^{58}$, qui détiennent en 1881 le pouvoir d'orienter dans un sens ou dans un autre la nouvelle composition française ? Tous sauf trois ont été élèves de l'École normale. Presque tous sont des lauréats de l'agrégation des lettres, où trois d'entre eux ont même été classés 
premiers. Seul Revillout, professeur à Montpellier, est agrégé d'histoire et géographie. La majorité d'entre eux (10 sur 16) a passé l'agrégation dans les années 1830 et 1840 ; la date moyenne de leurs agrégations est 1851, soit trente ans plus tôt. Le plus âgé, Philippe Roux, a passé le concours cinquante ans plus tôt; et il est professeur à la faculté de Bordeaux depuis sa création en 1839. Cinq sont par ailleurs doyens de leur faculté; soit le tiers des facultés des lettres de province. À s'en tenir à ces caractéristiques, ces hommes représentent bien l'Université la plus traditionnelle et la plus titrée, ce qui ne signifie pas forcément la plus passéiste ou la plus rétrograde car, en 1880, une très large fraction de l'Université est acquise au changement. D'autant que ces professeurs de faculté ont, des usages, des problèmes et des préoccupations de l'enseignement secondaire, une expérience qui est loin d'être négligeable. Tous, sans doute, y ont enseigné un moment après leur agrégation. Surtout, plus de la moitié d'entre eux y ont encore enseigné au cours des années 1870 qui ont été si décisives pour l'évolution des esprits et la transformation des programmes.

Il ne saurait être question ici d'étudier l'évolution de l'enseignement de la littérature française dans les facultés des lettres au cours des années 1870-1880, mais il n'est pas inutile de s'intéresser brièvement à quelques professeurs de la liste. Après le classicisme attardé qui a longtemps triomphé, sous la monarchie de Juillet et sous le Second Empire, avec les Villemain et les Nisard, des inconditionnels du XvII ${ }^{e}$ siècle, la tendance dans l'enseignement littéraire est désormais à l'ouverture, une ouverture prudente. Le grand recueil de morceaux choisis des lycées parisiens pendant cette décennie est celui d'un professeur de rhétorique parisien, Gustave Merlet ${ }^{59}$, qui moque, dans sa préface, "l'air un peu fané de tel gros bouquet cueilli jadis ${ }^{60} . .$. » et fait profession de « butiner dans toutes les provinces de notre littérature et [de] faire appel à toutes les facultés de l'intelligence ", jusque dans le XIX ${ }^{\mathrm{e}}$ siècle.

Plusieurs des professeurs des facultés des lettres abondent dans le même sens. Dans sa Leçon d'ouverture faite le 28 novembre 1870 à la faculté des lettres de Dijon où il a été nommé suppléant, Tivier ${ }^{61}$, évoquant l'image mutilée de la patrie, s'en prend aux thèmes favoris de la doctrine littéraire qui triomphait jusque-là, «ce fléau de la déclamation qui nous a fait tant de mal », «l'imitation à tout prix » et n'hésite pas à dénoncer l'étroitesse de vue non seulement de Boileau, mais de Villemain et de SainteBeuve qui n'ont rien compris au théâtre du Moyen Âge. D'autres, Philibert Soupé, dans Le Bien public de Dijon, ou Ernest Dugit à Grenoble ${ }^{62}$, prennent fermement position pour la circulaire contestée de Jules Simon. Dans un discours de rentrée des facultés à Clermont, Emmanuel Des Essarts appelle au "patriotisme littéraire " ${ }^{63}$, à "l'orgueil raisonné de ces chefs-d'œuvre qui, de siècle en siècle, ont fait resplendir la pensée française comme une flamme sur les hauteurs ». Et il se lance dans une comptabilité des "siècles de précellence", qui place la France en tête: "Nous avons à nous près de quatre siècles de précellence poétique contre deux siècles appartenant à l'Italie, un à l'Angleterre, un demi-siècle à l'Allemagne ${ }^{64}$ ". Pour la prose, nous avons toujours été les meilleurs. Sensibilité littéraire nouvelle, nationalisme littéraire poussé jusqu'au chauvinisme, conviction de la nécessité de réformer l'enseignement secondaire: ces professeurs de faculté qui vont fixer les sujets de composition française en 1881 sont en phase avec la société française de leur temps. Même un Charles Aubertin ne trouve plus aucune justification culturelle pour défendre la composition latine et se contente d'arguments bassement utilitaires. L'un de ces professeurs, Léon Moy, réunit le 8 mai 1881 la section de Douai de la Société pour l'étude des questions d'enseignement 
supérieur et s'attache avec ses collègues à fixer ce que doivent être les critères pour le choix des sujets de la nouvelle composition française du baccalauréat et pour l'évaluation des copies $^{65}$ : on anticipe déjà les dégâts que vont produire les sujets de pure mémoire, on place la cohérence du texte au rang de critère principal dans le jugement des copies et l'on préconise une forme de sujet qui permette d'estimer la « valeur intellectuelle » des candidats.

\section{Les sujets du baccalauréat de 1881}

Six semaines après avoir modifié le règlement du baccalauréat, Jules Ferry réforme en conséquence les programmes de l'enseignement secondaire ${ }^{66}$. Les professeurs de lettres des classes de troisième, seconde et rhétorique sont désormais officiellement chargés d'enseigner sous la forme d'un cours magistral l'histoire de la littérature française. Celui-ci est divisé en trois parties: quelques généralités en troisième («Notions d'histoire de la littérature française »), Moyen Âge et Renaissance en seconde ("Histoire sommaire de la littérature française jusqu'à la mort de Henri IV ») et, en rhétorique, "Histoire de la littérature française depuis l'avènement de Louis XIII ". Histoire de la littérature et histoire de la France (et de l'Europe) se trouvent maintenant logées à la même enseigne : pour l'une et pour l'autre il y a un cours magistral, des exercices de rédaction analogues, des programmes taillés dans les mêmes chronologies et au final une épreuve commune où elles figurent côte à côte dans l'arrêté de fondation.

41 En 1881, le baccalauréat n'a plus que deux sessions, où se présentent respectivement 7452 et 4395 candidats, soit un total de 11847 « examens », les candidats de la seconde session étant pour l'essentiel des recalés de la première. Les candidats passent toujours l'examen par "séries » de 20, mais la Sorbonne est autorisée à regrouper les séries et les facultés de province vont l'être bientôt également. Ont été retrouvés, pour les deux sessions de 1881, 248 sujets de composition française ${ }^{67}$. Ils peuvent être classés dans quatre séries générales :

- ceux qui font mention de la littérature française, ou de la littérature générale : 115 sujets $(46 \%)$

- ceux qui font mention de la littérature gréco-latine : 37 sujets (15\%)

- les sujets purement historiques : 84 sujets ( $34 \%)$

- les « divers » : 12 sujets (5\%). Par exemple, « En quoi consiste l'amour de la patrie? ?, ou « Discours de Guillaume Penn aux émigrants puritains venus en Amérique pour y fonder une société nouvelle (1682)».

La principale nouveauté, c'est la part que se taille d'emblée la composition historique, qui était à peu près totalement absente dans la composition latine ${ }^{68}:$ un sujet sur trois. Le programme d'histoire de troisième, mais surtout de seconde et de rhétorique est largement mis à contribution ${ }^{69}$. Par exemple : «Charlemagne et son temps. - Caractère de Charlemagne. Résultats des guerres qu'il a entreprises. Restauration de l'empire d'Occident. Influence de Charlemagne sur les lettres et les arts »; « Récit de la première croisade »; " Récit de la Saint-Barthélemy (24 août 1572) » ou "Situation générale de la France et de l'Europe à la mort de Richelieu ». L'histoire ancienne, qui est au programme des petites classes, n'est pas exclue pour autant : « Portrait de Périclès. A-til mérité de donner son nom à son siècle?"; "Qualis artifex pereo!70 Telle a été la dernière parole de Néron. Racontez brièvement la vie et la mort de cet histrion 
sinistre ». Mais, dans la grande majorité des cas, les sujets d'histoire sont des questions de cours, qui ont été traitées par le professeur de seconde ou de rhétorique, qui ont été étudiées, ou révisées, dans l'aide-mémoire ou dans le manuel et qui ont fait l'objet d'une « rédaction d'histoire ».

Les sujets littéraires sont au nombre de 152 (61\%). Première constatation : le passage du latin au français a entraîné une inversion de la proportion des sujets entre littérature française et littératures antiques. Les sujets portant sur la première sont désormais trois fois plus nombreux que les autres. Pourtant, le nouveau plan d'étude de 1880, qui officialise l'enseignement de l'histoire littéraire n'a nullement fait l'impasse sur les littératures anciennes. Les professeurs de lettres de troisième, seconde et rhétorique enseignent également, dans leur cours d'histoire, des « notions d'histoire de la littérature latine » et «de la littérature grecque ». Le renversement de tendance auquel on assiste en 1881, aux dépens des littératures anciennes, n'a donc pas été systématiquement organisé par le ministère. Ce sont bien les professeurs des facultés qui plébiscitent désormais la littérature française. Le changement de la langue véhiculaire en est évidemment le seul responsable. Du fait d'une conformité naturelle entre mode d'expression et contenu abordé, les habitudes universitaires avaient toujours poussé à traiter en latin des matières latines; et, avec ses 27 sujets de 1874 à 1880, la littérature française n'avait pu faire qu'une petite percée dans les sujets de composition latine. Maintenant que la langue libère les initiatives, c'est elle qui domine d'emblée.

Pour aborder des sujets de composition qui ont été proposés dans une époque passée, il est de bonne méthode de s'interroger non seulement sur la façon dont on les traiterait aujourd'hui (est-ce même bien nécessaire ?), mais surtout sur l'accueil (la " réception ", diraient certains) que ne pouvaient manquer de leur faire les élèves ou les candidats auxquels ils étaient destinés et sur la manière dont leur culture scolaire les amenait à les traiter. C'est ce critère qui sera mis en œuvre ici pour les 152 sujets littéraires proposés.

D'un bon nombre d'entre eux, on peut en dire autant que des sujets d'histoire : ce sont de pures et simples questions de cours, qui ont déjà été traitées par le professeur dans le cours d'histoire littéraire. Au moins 45 (soit $30 \%$ des sujets littéraires) sont du type : "Quels sont les historiens romains que vous connaissez? Appréciez celles de leurs œuvres que vous avez lues "; "Que savez-vous sur les pièces romaines de Corneille ?"; "Qu'appelle-t-on la Querelle des Anciens et des Modernes, et que savez-vous de cette histoire ? "; « Faire le tableau de la carrière littéraire de Bossuet »; « Tracer le tableau $\mathrm{du}$ progrès de la littérature française sous le règne de Louis XIII "; "Marquer les périodes de la littérature au XVII ${ }^{\mathrm{e}}$ siècle et en décrire les caractères généraux ». Tous sont des chapitres du cours, du manuel ou du mémento. Les candidats les apprennent par cœur et les régurgitent au moment de l'examen. Seule la mémoire est en jeu : il faut savoir sa leçon et l'on réussit sa composition, d'histoire ou d'histoire littéraire. C'est l'exercice de la « rédaction du cours » qui sert de modèle à cette épreuve.

Deuxième série de sujets littéraires, ceux qui portent sur la connaissance des œuvres et des personnages qui les animent : « Raconter l'histoire d'ÆEdipe et de sa famille d'après les trois tragédies de Sophocle: đedipe-Roi, Eedipe à Colone, Antigone»; analyse du Misanthrope, du Criton, de l'Oraison funèbre du prince de Condé; caractère de Don Diègue, de Sévère dans Polyeucte, rôle du Renard dans les fables de La Fontaine; théorie de Fénelon sur l'histoire, etc. Il y en a 25 , soit $16 \%$ des sujets littéraires. Le candidat n'y 
expose que la connaissance factuelle qu'il a des œuvres. Il s'est longuement exercé à l'analyse littéraire, on l'a vu, il sait résumer un texte, ou une œuvre, il sait même souvent en dégager les idées principales, le fil conducteur, les éléments essentiels de l'intrigue. L'analyse est sans doute l'un des exercices les plus féconds qu'ait utilisés la pédagogie ancienne du français. Mais la médaille a son revers. Dans bien des cas, l'œuvre n'a même pas été ouverte en classe et le maître, harcelé par le programme ou soucieux de protéger sa classe contre une littérature dévoyée, renvoie ses élèves vers des recueils d'" analyses ", ou leur dicte un résumé. L'analyse littéraire se dégrade alors en ersatz de l'œuvre, indispensable pourtant dans ce cadre historique ambitieux où trois littératures sont, dans leur intégralité, mises au programme de l'examen.

Le parallèle reste toujours aussi pratiqué (19 sujets, soit $12 \%$ ), comparaison entre deux personnages, deux œuvres, ou deux écrivains. Les parallèles de 1881 s'inscrivent exactement dans la lignée de ceux de la composition latine, par exemple «Comparer le rôle d'Émilie dans Cinna et celui de Chimène dans le Cid »; et ils en sont même parfois la traduction exacte : «Comparer le rôle de Narcisse dans Britannicus et celui de Mathan dans Athalie ». On imagine volontiers que l'exercice avait la faveur d'un bon nombre de maitres, comme une préparation utile à une réflexion plus élaborée sur la littérature. Certes, comme dans la série précédente, était essentiellement demandée, derrière un habillage comparatif, une connaissance factuelle des œuvres. Dans l'échelle des exercices permettant de mener l'élève à une réflexion littéraire de qualité, il se situait pourtant un degré au-dessus, puisqu'il obligeait à mettre en relation deux tragédies ou deux personnages, dont il était difficile de parler si l'on n'en avait pas une connaissance directe. Mais c'était plutôt, de par son caractère propédeutique, un exercice de la classe de seconde.

Une quinzaine de sujets (soit $10 \%$ du total), annoncent la future dissertation littéraire. Mais comment les élèves de l'époque pouvaient-ils traiter des sujets comme: «Développer cette pensée d'un de nos poètes: Le vers se sent toujours des bassesses du cœur", "Qu'entend-on lorsqu'on dit que les personnages de Corneille raisonnent trop?", ou "Molière est-il un moraliste ? Rappelez quelques-uns des traits de son théâtre qui nous peuvent autoriser à l'appeler de ce nom »? De deux choses l'une : ou le professeur a traité ces questions dans son cours, et l'exercice sollicite chez le candidat une mémoire qu'il faut supposer lourdement chargée; ou l'élève est renvoyé à sa connaissance (et à la mémorisation) des œuvres, à la lecture et à l'explication qui en est faite en classe, et il bute alors sur une difficulté probablement insurmontable. On aborde ici un problème qui n'a pas été évoqué ci-dessus, mais qui va rapidement devenir central dans le débat qui s'ouvre en 1881 : l'enseignement du français doit-il viser à enseigner aux lycéens et aux collégiens l'histoire de la littérature et le résumé de ses chefs-d'œuvre, ou doit-il se fixer pour fin de les mettre en contact avec l'œuvre littéraire, quelle qu'elle soit (d'où la nécessaire extension des programmes jusqu'au XIx ${ }^{e}$ siècle), de former en eux un sens de la littérature, d'affiner le goût pour la phrase et le texte et de contribuer à développer leur plaisir de lecteur? C'est, on le sait, Gustave Lanson qui posera ce problème dans toute sa force et qui fera admettre un peu plus tard la totale inutilité de l'enseignement dogmatique de l'histoire littéraire pour le secondaire ${ }^{71}$.

On n'en est pas là en 1881. On pense même exactement le contraire. C'est une débauche d'érudition littéraire qui s'abat sur les classes, aux dépens de la lecture des textes. L'explication de textes qui prévaudra au $\mathrm{xx}^{\mathrm{e}}$ siècle n'est encore qu'en formation. Parmi 
ses précurseurs, on cite Charles Thurot, dans son enseignement à l'École normale des années 1870, on cite les manuels d'Augustin Gazier (1880) et de Gustave Allais (1884); bientôt viendront les campagnes de Brunot et de Lanson; enfin la réforme de 1902. Le professeur de 1880, qui est parfaitement sensible à la beauté littéraire, qui est en mesure de la faire ressentir par sa classe, n'est pas encore capable d'apprendre à l'élève à extraire méthodiquement de l'œuvre les éléments qui permettraient de construire une réponse circonstanciée à la plupart des questions posées sous forme de "dissertations littéraires ", à utiliser les données apprises, à les discuter et à apporter sur elles et sur la question soumise à examen un point de vue personnel. À peine créé, l'enseignement secondaire féminin l'emporte déjà sur le vieil enseignement classique masculin pour ce qui est du contact avec les textes français : "Presque partout, la lecture et la récitation sont déplorables [...]. Combien, sur ce point important des études, les lycées et collèges de jeunes filles nous sont supérieurs ! ", écrit l'inspecteur général Deltour ${ }^{72}$.

De toute façon, faute d'expérience en la matière, les jurys, habitués aux épreuves de la licence, visent trop haut et confondent le baccalauréat et les concours de recrutement. On surprend ainsi le vieux professeur de Bordeaux, Philippe Roux, à donner à des élèves de rhétorique le même sujet qu'il avait déjà donné trente ans plus tôt (en 1852) à des candidats à la licence dans la même faculté : «Qu'a voulu dire Horace, dans son Art poétique, lorsqu'il a comparé l'une à l'autre la poésie et la peinture, Ut pictura, poesis ? ${ }^{73}$ ». Sujet qui dépasse évidemment les compétences de ceux qui ne l'auraient pas déjà traité en classe et qui ne reproduiraient pas le corrigé. Et que penser du sujet de dissertation donné en 1868 à l'agrégation des lettres, "Montrer que Britannicus est la pièce des connaisseurs" et sur lequel doivent plancher les candidats bacheliers de 1881, à Caen et à Paris? Que peut raisonnablement dire sur la question un élève de rhétorique, même excellent, qui n'aurait comme bagage qu'une bonne connaissance des pièces de Racine?

Dernière série ${ }^{74}$ : une quarantaine de sujets littéraires (soit $26 \%$ ) doivent être traités sous forme de lettre (exceptionnellement de discours, 4 , soit $3 \%$ ) : untel écrit à untel pour lui décrire un tableau, pour lui raconter une scène, pour lui dire que..., pour lui demander si..., pour le convaincre de... La plupart d'entre eux reposent sur les mêmes bases que les sujets précédents : connaissance du cours ou des œuvres et des auteurs du programme. Par exemple, «Lettre de Boileau à Racine sur la mort de Molière (février 1673). Il a besoin d'épancher sa douleur dans le sein d'un ami : Molière est mort ! Il faut oublier de misérables querelles devant cette tombe. Éloge de Molière. La scène française a fait une perte irréparable». Il s'agira évidemment pour le candidat de montrer sa connaissance de l'œuvre de Molière, de sa brouille avec Racine, mais les qualités rhétoriques qu'il peut mettre en œuvre et l'émotion qu'il peut tenter de faire partager au correcteur visent à compenser les défaillances ou les lacunes dans les souvenirs. On retombe dans le modèle rhétorique ancien.

Permettons-nous ici un peu d'anticipation, pour cadrer plus utilement les quelques remarques conclusives qui s'imposent et projetons-nous un instant en 1925 : que seront devenus à cette date les pourcentages des différentes classes de sujets donnés au baccalauréat ? En 1925, l'histoire aura quasiment disparu : 3 \% des sujets. La littérature ancienne aura subi le même sort: 3 \% également. C'est la littérature française, avec $80 \%$ des sujets, qui écrasera tous ses concurrents ${ }^{75}$. Quand on prend en considération les pourcentages des mêmes séries au cours de la période intermédiaire 1881-1925, on 
constate une évolution relativement lente jusqu'en 1900 suivie d'une forte accélération dans le premier quart du vingtième siècle. C'est donc bien la littérature française qui est l'avenir de la composition française du baccalauréat: on s'en doutait un peu. À partir de 1881, le sort du baccalauréat ès lettres est donc lié étroitement à celui de l'enseignement du français et des grands textes de la littérature nationale.

Mais où en sommes-nous après les deux sessions de 1881? La nouvelle épreuve de composition française de littérature, solidement appuyée sur trois années de cours d'histoire littéraire, transforme l'examen en un impressionnant système de contrôle des connaissances littéraires des élèves. Les exercices de composition qui servent désormais de modèles, la rédaction du cours et l'analyse littéraire, sont des genres mineurs, utiles certes, mais à condition d'être mis au service d'une formation plus exigeante. Deux grands exercices sont en compétition pour constituer la clé de voûte des études classiques, le discours et la dissertation. Le premier, trop lié à la rhétorique, a perdu la partie après au moins deux siècles de domination exclusive ; il n'offre plus, dans notre corpus de sujets, qu'un cadre désuet pour une mise en forme peu adaptée à des développements littéraires. Le second a tenté, en 1881, son entrée dans le baccalauréat. Et, pour le moment, c'est un échec. La dissertation a derrière elle une carrière moins longue que celle du discours, mais elle a fait ses preuves lorsqu'elle était proposée à des candidats plus âgés et mieux formés. Plus récemment, elle a réussi à s'adapter à la classe de philosophie : il y a depuis 1866 une épreuve de dissertation philosophique au baccalauréat. L'épreuve peut-elle descendre encore d'une année dans le cursus des études? Le discours étant désormais hors jeu, la dissertation peut-elle à son tour devenir l'exercice majeur de la classe de rhétorique sans se dégrader systématiquement en rédaction du cours ou en analyse littéraire? Tel est l'enjeu devant lequel se trouve désormais placée l'Université.

Car ce ne sont plus seulement les humanités classiques qui sont en cause dans la crise où s'enfonce le baccalauréat, c'est l'esprit même de cet enseignement secondaire, fondé sur la lecture, sur l'explication des textes, sur la réflexion personnelle, sur la composition de textes par l'élève. La dissertation est un pari ambitieux pour des élèves de rhétorique (on dira bientôt la "première ») qui sont encore insuffisamment préparés à relever le défi. Mais il n'y a pas d'autre alternative : il faut soit revenir à l'ancienne rhétorique dont on ne veut plus, soit se lancer dans une nouvelle culture où le français primera définitivement sur le latin, où la lecture attentive des textes remplacera les résumés d'histoire littéraire et où l'expression de la pensée personnelle de l'élève se substituera aux fleurs de la rhétorique. Car pour le corps enseignant dans son ensemble, pour l'opinion publique elle-même, la formation secondaire doit avoir pour but non pas l'érudition historique mais l'acquisition d'une culture générale. La nouvelle voie qui s'ouvre à partir de 1881 suscite donc un malaise considérable. Tel qu'il est, le baccalauréat risque de remettre en cause la fonction même du secondaire, et certains en viennent à envisager sa suppression.

L'un des résultats les plus spectaculaires de cette crise, c'est le développement du parascolaire. Les boîtes à bac ne datent pas de 1881 : elles étaient déjà actives vingt ans plus tôt. Mais l'examen n'a cessé de proliférer et, en particulier, les épreuves écrites se sont multipliées, à un rythme décennal : une en 1841 ; deux en 1853 ; trois en 1865 ; quatre (sur deux années) en 1874 ; cinq en 1881. La machine docimologique s'emballe; et le secteur privé s'engouffre dans la brèche du marché du baccalauréat où il réalise des profits importants. Voici, par exemple, la maison Croville-Morant, qui s'est 
installée au 20, rue de la Sorbonne. Elle propose aux candidats une préparation efficace et ciblée aux différentes épreuves écrites de l'examen. Depuis 1878, elle publie en période d'examen une feuille quotidienne petit format, Épreuves écrites et orales $d u$ baccalauréat ès lettres (Examens de la Sorbonne) ${ }^{76}$. Y paraissent chaque jour les textes des épreuves, la liste des candidats, des reçus, les noms des membres du jury, les questions qu'ils ont posées à l'oral, etc., etc. Les collaborateurs de cette officine recueillent ainsi tous les sujets proposés, se font communiquer ceux de province, publient des répertoires ${ }^{77}$ rétrospectifs des sujets parus dans la feuille quotidienne, avec les corrigés types, d'abord la traduction des versions latines, plus tard les corrigés des sujets de composition française; ils proposent bientôt des cours de rattrapage, des cours de vacances « avec des professeurs agrégés ».

Le bachotage est roi et rares sont les élèves qui y échappent, tant se fait sentir la nécessité des manuels pratiques et des aide-mémoire. L'Université est en passe de se faire déposséder du baccalauréat par l'entreprise privée. Charles Bigot dresse, à la veille $\mathrm{du}$ passage à la composition française, un vivant tableau de cette nouvelle profession qui s'installe en marge de l'Université : "Une branche d'industrie nouvelle a surgi parmi les professions parisiennes; à côté du chef d'institution marchand de soupe qu'avaient peint les satiriques de la génération précédente et aux spéculations duquel la loi de 1850 et la concurrence des pensionnats ecclésiastiques avaient porté un coup fatal, on a vu apparaître " l'entraîneur » aux examens du baccalauréat. Il a prospéré, il a fait école. Son industrie s'est élevée à la hauteur d'une institution sociale. Tel de ces messieurs peut se vanter d'avoir fait, à lui seul, réussir plus de cancres que le lycée Louis-le-Grand n'a fait réussir de bons élèves. Le préparateur au baccalauréat a été dans les trente dernières années le plus grand bienfaiteur de nos classes dirigeantes... " $^{78}$.

Pendant plusieurs années, le ministère joue la montre. Le temps travaille pour lui : il escompte en effet engranger à terme, au profit de la composition française du baccalauréat, le bénéfice de la réforme des études secondaires, lancée par le nouveau plan d'études de 1880. Mieux préparés à la composition française tout au long de leur scolarité, les élèves doivent pouvoir aborder l'épreuve littéraire dans des conditions plus satisfaisantes. Mais de nouvelles mesures s'avéreront vite indispensables et le ministère doit corriger le tir. Dès 1885 , une enquête lancée ${ }^{79}$ par le ministre Fallières auprès de tous les établissements pose aux professeurs et à l'administration locale le problème de l'examen, et donne la mesure des profonds changements réclamés dans toutes les académies. Il faudra donc assouplir le système en donnant trois sujets au choix pour tenir compte des différentes aptitudes des élèves (1891), rassembler autant que possible les candidats dans une série unique pour éviter les injustices, associer les professeurs des lycées à la correction des épreuves, etc. Simultanément, les élèves de l'enseignement spécial voient leurs études couronnées par un baccalauréat (1883); un rapprochement s'opère à partir de 1891 et un certain nombre de facultés vont même donner des sujets de composition française identiques pour les classiques et pour les modernes.

58 Mais c'est surtout avec une transformation des programmes et des méthodes de l'enseignement $d u$ français que l'Université reprendra la main. Des circulaires ministérielles encouragent maintenant la «lecture libre», si longtemps tenue en laisse, et luttent contre les excès de la rédaction du cours ${ }^{80}$. Le cours d'histoire littéraire, dénoncé dès le début par bien des maîtres, par Lanson, par l'inspection générale, est supprimé en 1902. Les programmes d'auteurs se modernisent. Surtout une nouvelle 
discipline se met en place, l'explication de texte français qui devient à partir de 1902 le principal exercice de la classe, de toutes les classes. En quelques années, comme le dit une instruction officielle de 1909, elle " a passé du second plan au premier et devient de plus en plus, dans sa précision nouvelle, un instrument d'éducation intellectuelle et morale $»^{81}$. C'est sur elle que l'on compte pour faire de la dissertation littéraire la clé de voûte d'une nouvelle discipline.

Alors, quel rôle attribuer au baccalauréat dans la création de la dissertation littéraire à la fin du XIX ${ }^{e}$ siècle? Le lent déclin des humanités classiques au cours de l'histoire moderne et contemporaine connaît enfin son heure de vérité, la composition française se substitue définitivement au discours latin, et les arrêtés de 1880 réformant le baccalauréat et le plan d'études ne sont que la conséquence de cette évolution. Mais grâce à l'examen, une expérience considérable, à caractère didactique, pédagogique et culturel, s'accumule en quelques années dans l'instruction publique. Fonctionnant comme un formidable accélérateur des prises de conscience, le baccalauréat, autour duquel se concentrent les innovations, les déboires, les problèmes et les conflits, met à jour un certain nombre d'idées nouvelles, qui, sans son intermédiaire (il n'est pas hors de propos d'imaginer une suppression du baccalauréat puisque le ministère lui-même l'envisage en 1885), auraient probablement exigé de nombreuses décennies avant de s'imposer. Il n'y a probablement pas d'autre époque de l'histoire où les esprits aient collectivement évolué aussi vite sur des questions d'enseignement. Dressons une liste non exhaustive, et qui mériterait d'ailleurs d'être elle-même démultipliée, de ces idées nouvelles, en suivant approximativement la chronologie des événements :

- c'est la littérature française qui doit être au centre des études littéraires; l'enseignement ex cathedra de l'histoire littéraire est la solution du problème (années 1870, début des années 1880) ; l'enseignement ex cathedra de l'histoire littéraire n'est pas la solution du problème (ensuite); - les exercices habituels des classes (rédaction du cours, analyse des œuvres) ne sauraient offrir une alternative satisfaisante au vieux discours latin ; - la lecture des grands textes de notre littérature, y compris les plus récents, doit être l'activité principale des classes littéraires du secondaire ; - un nouvel exercice doit être installé dans les classes pour traiter oralement les textes français ; un nouvel exercice écrit de composition en français s'impose comme l'objectif essentiel de l'enseignement secondaire ; - il doit être préparé tout au long de la scolarité par une progression d'exercices de composition; - la mise en place de cet exercice n'est possible qu'en relation avec le nouveau modèle d'explication des textes qui doit avoir cours dans les lycées et les collèges ; - l'enseignement secondaire, qui est le berceau de cette nouvelle culture, doit se voir confier un rôle important dans le déroulement du baccalauréat; - la disparition de la composition latine au baccalauréat plaçant les classiques et les modernes dans des conditions analogues, les mêmes épreuves pouvant être données aux uns et aux autres, pourquoi ne pas envisager alors un baccalauréat unifié ? (etc.)

61 Toutes ces idées sont des idées neuves, qui apparaissent (et parfois disparaissent) au cours de la période, bouleversant les certitudes anciennes et les hiérarchies les mieux établies, et qui vont s'imposer aux esprits en vingt ou trente ans. C'est au baccalauréat qu'on doit la rapidité de cette mutation. 


\section{NOTES}

1. L'ouvrage de J.-B. Piobetta : Le Baccalauréat de l'enseignement secondaire (1937) n'a pas encore été remplacé, et il reste la source indispensable pour l'histoire du baccalauréat, en particulier pour la réglementation. Les sujets de composition française donnés à l'examen sont reproduits dans notre recueil La Composition française au XIXe siècle, dans les principaux concours et examens, de l'agrégation au baccalauréat (Paris, Vuibert, INRP, 1999). Martine Jey a consacré une partie de sa thèse de 1996 à l'étude et au classement des sujets donnés au baccalauréat (période 1881-1896) ; cf. La Littérature au lycée : invention d'une discipline (1880-1925), Paris, 1998.

2. Respectivement, règlements du baccalauréat du 3 août 1857 , du 28 novembre 1864 et du 25 juillet 1874.

3. Cf. Louis Petit de Julleville: «Le jury du baccalauréat ès lettres", Revue internationale de l'enseignement, 1881, I, pp. 346-358.

4. « Il est nécessairement appelé à donner son avis », dit la loi du 15 mars 1850 (loi Falloux).

5. Émile Blanchard : "L'Instruction générale en France. L'observation et l'expérience », Revue des Deux Mondes, 15 octobre 1871.

6. Cf. Antoine Prost : Histoire de l'enseignement en France, 1800-1967, Paris, Colin, 1968, p. 45.

7. Dès novembre 1881, la faculté de Douai donne comme sujet de composition: "Comparaison entre le Misanthrope et le Tartuffe. Dites laquelle vous préférez de ces deux comédies de Molière, et pourquoi ». La même année, la faculté de Poitiers donne le sujet: «Nicole, voyageant en Allemagne, publia une traduction latine des Provinciales sous le pseudonyme de Wendrockius. Arnauld lui écrit pour le remercier et le féliciter. Par ce moyen toute l'Europe savante pourra connaître et apprécier les Petites Lettres ». Les établissements catholiques ne peuvent plus se permettre de faire l'impasse sur ces œuvres : ils se tirent d'affaire en dictant à leurs rhétoriciens des « analyses » toutes prêtes qui leur évitent la lecture de l'œuvre elle-même, ou en utilisant les éditions « épurées » de l'abbé Lejeune ou de l'abbé Figuière qui réduisent à vingt ou trente vers la grande scène de l'acte IV de Tartuffe.

8. La hiérarchie catholique lance en 1882 un nouveau périodique, L'Enseignement chrétien, dont l'une des fonctions est de faciliter le recyclage de ses professeurs en direction du nouveau baccalauréat.

9. Arrêté du $1^{\mathrm{er}}$ août 1843 décidant l'attribution de prix de récitation dans les collèges.

10. Il comporte aussi, depuis 1865 , une «composition française sur un sujet de philosophie» (Règlement du28 novembre 1864), et, depuis 1874, «la traduction, en français, d'un texte de langue vivante » à l'écrit de la seconde partie.

11. Cf. André Chervel : La Culture scolaire. Une approche historique, Paris, Belin, 1998, chap. II.

12. On disait encore, il y a quarante ou cinquante ans, "faire sa préparation ».

13. Mentionnée dans les réponses à une enquête ministérielle de 1872.

14. On dit aussi, dans ce sens, un « extrait».

15. Arrêté sur le plan d'étude des lycées (23 juillet 1874).

16. Louis Petit de Julleville : Le Discours français et la dissertation française, Paris, 1868, p. 1.

17. Cf. La Composition française au XIX siècle, op. cit., chap. V.

18. Cf. Pierre Albertini : L'Enseignement classique à travers les exercices manuscrits des élèves, 1600-1940 (INRP, Collection Rapports de recherches, 1986, n 5), réalisé essentiellement à partir du Catalogue général des manuscrits des bibliothèques de province. Le cahier de cours de rhétorique le plus récent mentionné dans ce répertoire date de 1835.

19. Règlement du baccalauréat ès lettresdu 5 septembre 1852 .

20. Les règlements de 1857 et de 1864 répètent la même formule. Celui de 1874 (Décret sur le baccalauréat ès lettres du 25 juillet 1874) opère une petite régression en imposant aux candidats de 
la classe de rhétorique des «interrogations [...] sur les principales notions de rhétorique et de littérature classique ».

21. Op. cit., p. 3.

22. Les textes d'origine ministérielle présentés ici sont empruntés au Bulletin administratif du ministère de l'Instruction publique et au recueil de Circulaires et instructions relatives à l'Instruction publique publié par Delalain.

23. Mgr Dupanloup jette toute son autorité dans la balance (Seconde lettre de M. l'évêque d'Orléans aux supérieurs et professeurs de ses petits séminaires), accusant Jules Simon de détruire les études classiques et défendant la composition latine: «En latin, l'entrave de la langue empêche l'imagination de divaguer : en français, l'imagination est trop à son aise ». Le P. Sengler parle de « coup d'État» (Un Coup d'État dans l'enseignement secondaire en France. Le Coup d'État de M. Jules Simon, Lille, 1873), et de "coup de grâce donné aux études classiques ». Voir aussi une lettre célèbre de Cuvillier-Fleury au Journal des débats (30 octobre 1872), reproduite dans La Réforme universitaire (1873). Mais de nombreuses voix s'élèvent pour approuver les mesures préconisées par Jules Simon; la plus célèbre est celle du linguiste Michel Bréal (Quelques mots sur l'instruction publique en France, Paris, Hachette, 1872).

24. Archives nationales, Circulaires rectorales de l'académie de Paris, AJ/16/2510. Reproduit dans [Conseil académique de Paris] Exposés de M. Ad. Mourier, sessions 1862-1878, Paris, Delalain, 1879, pp. 366-378.

25. Le plan d'études qui régit les études secondaires jusqu'en 1874 est celui du 30 août 1852, à peine modifié par Duruy en 1865 : l'enseignement littéraire de la classe de rhétorique ne comporte que des " notions élémentaires de rhétorique et de littérature ».

26. A.N., F/17/6872/2, « Révision des programmes, 1869-1875».

27. La première partie a également un oral, qui comporte des « explications... d'auteurs français, grecs et latins " et des "questions d'histoire, de géographie, de rhétorique et de littérature classique ».

28. Pour la « composition française sur un sujet de philosophie », voir ci-dessus, note 10.

29. Plus de 12000 copies de version latine du baccalauréat des années 1841-1847 sont conservées aux Archives nationales, mais il n'a été jusqu'à présent découvert que fort peu de copies de la deuxième moitié du siècle. Mentionnons cependant une vingtaine de copies de composition française, de composition latine et de version latine du baccalauréat ès lettres de la faculté de Douai (1857), conservées aux Archives départementales du Nord. Je remercie M. Philippe Marchand qui a découvert ce fonds et m'a communiqué l'information. cf. son étude, infra, pp. 201-208.

30. Pour la période précédant 1880, il s'agit essentiellement des périodiques suivants: Le Conseiller de l'enseignement public, L'Instruction publique et la Revue de l'instruction publique.

31. Pour la période 1853-1870. À Alger est instituée une "école supérieure des lettres » qui est habilitée à faire passer le baccalauréat à partir de juillet 1880. On ne connaît aucun sujet algérois avant 1887.

32. Recueil de versions latines et de discours latins donnés à la faculté des lettres de Toulouse, Périgueux, Dupont, 1872.

33. Caractère de Polyeucte dans la tragédie de Corneille.

34. Mardochée s'adresse à Esther qui, craignant pour sa vie, hésite à aller supplier le roi Assuérus en faveur des Juifs. Autrement dit: traduire en latin des morceaux de la tirade de Mardochée (acte 1, scène 4) que beaucoup de candidats connaissent par cœur.

35. Vous comparerez, dans Racine, le comportement, le caractère et le personnage d'Athalie et d'Agrippine.

36. Si tant est que les lacunes de notre documentation autorisent ce genre d'affirmation.

37. cf. La Fontaine, sources et postérité d'Esope à l'Oulipo, éd. établie et présentée par André Versaille, Bruxelles, éd. Complexe, 1995. 
38. Fortoul l'avait rebaptisée la classe de « logique ».

39. Comparer Mathan dans Athalie et Narcisse dans Britannicus; opposer, chez Racine, les personnages de Burrhus et de Narcisse.

40. Quels sont les principaux poètes épiques chez les anciens et chez les modernes?

41. À une exception près, un sujet de discours latin donné à Montpellier en août 1880, tous les sujets retrouvés pour la période qui va de la session de juillet-août 1874 à celle d'octobrenovembre 1880 , sont parisiens. Les lacunes importantes de notre documentation biaisent un peu cette analyse. Mais l'exemple de Douai montre que certaines facultés de province ne sont pas hostiles à ce type de sujet.

42. Le gouverneur d'Oreste, déguisé en messager, annonce faussement à Clytemnestre qu'Oreste est mort lors des Jeux pythiques.

43. Raconter les voyages d'Enée après la chute de Troie.

44. Comparer la vie et la mort de Démosthène et de Cicéron; comparer Enée et Turnus chez Virgile.

45. Brève présentation de l'Aulularia de Plaute; exposé rapide des douze chants et des personnages de l'Enéide; le Philoctète de Sophocle : sujet, personnages, dénouement.

46. Essai sur Tite-Live, Paris, Hachette, 1856.

47. Le sujet de 1829 n'a pas été retrouvé.

48. Donner les principales raisons de la corruption de la langue littéraire à Rome après le règne d'Auguste. Un seul sujet, donné en 1831, fait référence aux historiens modernes, mais pour mieux louer ceux de l'Antiquité: Cur et quomodo veterum ingenia recentiorum ingeniis in historia conscribenda praecelluerunt? «Pourquoi et en quoi, dans l'histoire, les Anciens l'emportent-ils sur les Modernes?»

49. Il fait date dans l'histoire de l'enseignement : c'est la première fois qu'un sujet de dissertation littéraire (au sens où nous l'entendons aujourd'hui) est donné à un examen public en France.

50. Arrêté du 28 février 1837.

51. La France a acquis autant de gloire par sa littérature que par ses conquêtes.

52. Comparer Virgile et Racine.

53. Montrer qu'avec Andromaque, Racine a créé un nouveau type de tragédie qu'un éminent critique a pu baptiser « tragédie franco-grecque ».

54. Paul Janet : « Les réformes dans l'enseignement », Revue des Deux Mondes, 15 nov. 1872.

55. Compositions littérairesfrançaises et latines sur les sujets dictés par les facultés dans les examens du baccalauréat ès lettres, avec des conseils, des préceptes, des applications et des développements, Paris,

$1854 ; 3^{\mathrm{e}}$ éd., 1878, Compositions latines; 4 édition, revue, corrigée et augmentée, Compositions françaises, 1882 .

56. Ils sont recueillis dans Épreuves écrites et orales du baccalauréat ès lettres (Examens de la Sorbonne), publiées par la librairie A. Morant, Croville-Morant, gendre et successeur.

57. Le classement dans ces deux catégories ne peut s'opérer sans une part d'arbitraire, et il en va de même pour tous les classements qui ont été opérés jusqu'ici. Le sujet «Un député des Arcadiens à Platon. - Arcadum legatus Platonem orat ut in regionem suam venire velit novae civitati Megalopoli leges scripturus " (un député des Arcadiens supplie Platon de venir dans son pays rédiger les lois d'une nouvelle cité, Mégalopolis), donné le 28 octobre, a été classé parmi les sujets littéraires à cause de Platon, mais le sujet «Miltiade aux Athéniens (qui vont engager la bataille de Marathon). - Oratio Miltiadis ad milites Athenienses apud Marathona pugnaturos ", qui est le dernier sujet de composition latine proposé au baccalauréat (le 9 décembre 1880), est classé dans les discours faute de mentionner le nom d'un écrivain.

58. Écartons l'école supérieure des lettres d'Alger, pour laquelle aucun sujet n'a été retrouvé. À la Sorbonne, l'enseignement de la littérature française est depuis 1810 partagé entre deux chaires, l'une de poésie, l'autre d'éloquence. Le titulaire de la chaire de poésie, Charles Lenient, qui a été élu député, est suppléé par un professeur de Louis-le-Grand, Léopold Marcou. 
59. Extraits des classiques français, $\mathrm{XVII}^{e}, \mathrm{XVIII}^{e}$ et XIX $\mathrm{X}^{e}$ siècles, Paris, 1871.

60. L'allusion vise les Leçons françaises de littérature et de morale (1804) de Noël et Delaplace, rééditées jusqu'en 1862 .

61. Il sera nommé en 1873 à Besançon.

62. Projet d'une réforme dans les études classiques. Discours de réception prononcé le 27 décembre 1872. Dugit vient d'être chargé du cours de littérature française à la faculté de Grenoble.

63. Des Essarts (E.) : Du patriotisme littéraire, 1876.

64. À cause de Goethe et Schiller, il accorde à l'Allemagne la fin du XVIII ${ }^{\mathrm{e}}$ siècle et le début du XIX $\mathrm{X}^{\mathrm{e}}$.

65. (Léon) Moy: "Quelle doit être la nature de la composition française au baccalauréat ès lettres? ", Revue internationale de l'enseignement, 1881, II, pp. 182-184.

66. Plan d'études et programmes de l'enseignement secondaire classique (2 août 1880).

67. Le total réel des sujets proposés en 1881 est impossible à chiffrer : probablement le double. Outre les copies, qui ont toutes disparu, manquent également aujourd'hui les rapports des jurys, dont on imagine aisément qu'ils ont dû être étudiés minutieusement par la direction de l'Enseignement secondaire et qu'ils ont pu prendre ensuite une autre direction que celle des Archives nationales. On trouvera le libellé des sujets dans La Composition française au XIX siècle, op. cit., pp. 303 sq.

68. Quelques exceptions cependant : par exemple « De l'ostracisme », proposé à Paris en 1876.

69. Sur ce point aussi, répartir les sujets entre les deux catégories «littérature » et " histoire » implique qu'on s'est fixé des règles relativement arbitraires, tellement les deux disciplines sont intimement liées dans nombre de cas, en particulier dans tous les sujets portant sur « le règne de Louis XIV ».

70. Quel artiste meurt en moi! (Suétone, Néron, 49, 1).

71. "L'Étude des auteurs français dans les classes de lettres ", Revue universitaire, 1893, tome 2, pp. 255-271. Voir aussi son avant-propos à l'Histoire de la littérature française.

72. Rapport du 4 juillet 1884 (A.N., F/17/6874).

73. Les poésies sont comme les peintures. Le sujet proposé à la licence en juillet 1852 était libellé ainsi : "Que faut-il penser de l'application absolue qu'on a faite à la poésie du mot d'Horace : Ut pictura poesis? La poésie n'est-elle en effet qu'une peinture parlante, une imitation harmonieuse ou bien dérive-t-elle aussi, par ses genres primitifs, d'un autre principe?»

74. Quatre sujets sur les 152 n'ont pu être classés dans aucune des séries ici présentées et discutées: un portrait, une narration et deux explications de textes de La Fontaine, qui demandent au candidat un peu plus que l'analyse traditionnelle.

75. Il reste en $192513 \%$ de sujets généraux, moraux, patriotiques, réflexions sur les voyages, la langue, l'homme d'action, etc.

76. Le titre devient, en 1885, Journal des examens de la Sorbonne, baccalauréats [...]. Partie comprenant les épreuves écrites.

77. La plupart des auteurs de recueils de sujets et de corrigés de l'époque sont soit des collaborateurs de la maison Croville-Morant (Jean-Baptiste Béraud, Henri Carle, Victor Derély, Gasc-Desfossés, Edmond Guyot, Édouard Jullien, Auguste Marais, Georges Meunier, les frères Navatel, Léon Pajot, Bernard Pérez, Tempestini) soit des membres de l'enseignement catholique, soucieux de ne pas se laisser distancer par une évolution dont ils ont perdu le contrôle (Joseph Brugerette, abbé James Condamin, Mgr Théodore Delmont, abbé H. Rigault, abbé S. Verret).

78. Bigot (Charles), «La réforme des examens", Revue politique et littéraire (alias Revue bleue), juillet-décembre 1880.

79. Circulaire du 10 février 1885.

80. «Que [MM. les professeurs] exigent de simples résumés, au lieu de longues rédactions » (Circulaire sur l'application du plan d'études dans les lycées et collèges, 4 novembre 1882) ; «La rédaction [d'histoire] n'est que la copie de notes prises en classe ou la reproduction d'un livre [...]. Cet exercice, qui est déjà, du reste, en partie abandonné, doit donc être absolument 
proscrit » (Lettre aux membres du personnel administratif et enseignant des lycées et collèges, 15 juillet 1890).

81. Instruction relative à l'enseignement du français dans les lycées et collèges (22 février 1909).

\section{RÉSUMÉS}

En juillet 1881 s'ouvre la première session du nouveau baccalauréat ès lettres réformé par Jules Ferry. La composition latine est supprimée et les candidats doivent rédiger une " composition française sur un sujet de littérature ou d'histoire ». C'est un tournant majeur dans l'histoire des humanités classiques. Si la mesure a bien été préparée dès 1880 par les nouveaux programmes, l'enseignement secondaire n'a pas encore élaboré le nouvel exercice scolaire qu'on baptisera plus tard la «dissertation littéraire ». Les sujets proposés aux candidats en 1881 et les années suivantes restent proches des exercices traditionnels que sont l'analyse, le discours ou la rédaction du cours. Il faudra une seconde réforme, vingt ans plus tard, pour asseoir solidement la dissertation de la classe de première sur les nouvelles méthodes de l'explication de texte. L'article s'attache à montrer dans quelles conditions le discours latin a cédé la place à la composition française, pourquoi le baccalauréat subit alors une crise profonde, et les solutions qui se sont imposées d'elles-mêmes.

The first session of the new Arts baccalauréat overhauled by Ferry began in July 1881.The Latin composition was suppressed and candidates had to write a 'French composition on a subject of literature or history'. This was a major turning point in the history of the humanities. Though the measure adequately fitted into the new curriculum as early as 1880 , the secondary education system had not yet initiated the new school exercise which was later called the "literary essay". The examination questions put to the candidates in 1881 and in the following years remained close to such traditional exercises as analysis, composition and lesson tests. A second reform was necessary, some twenty years later to firmly base the essay of the class of première* on the new methods of textual analysis. The article attempts to show in which conditions the Latin composition gave way to the French essay, why the baccalauréat found itself in a real predicament, and the self-explanatory solutions chosen at the time.

Im Juli 1881 wird das neue von Jules Ferry reformierte literarische Abitur erstmals abgenommen. Der Aufsatz in lateinischer Sprache ist weggefallen, und die Kandidaten müssen stattdessen eine entscheidende Neuerung in der Geschichte der klassischen humanistischen Bildung - einen Aufsatz in französischer Sprache über eine Thema aus der Literatur oder der Geschichte anfertigen. Zwar sind im Rahmen dieser Neuerung seit 1880 neue Unterrichtsprogramme eingeführt worden, doch hat sich im Unterricht selbst die neue Übung, die man später als „Literaturaufsatz“ bezeichnen wird, noch nicht entwickelt. Die Themen, die den Kandidaten 1881 und in den folgenden Jahren zur Bearbeitung angeboten werden, haben sich noch kaum von den traditionellen Übungen (Analyse, Abhandlung über oder Zusammenfassung einer Unterrichtseinheit) entfernt. Es war vielmehr 20 Jahre später eine zweite Reform notwendig, um die neuen Methoden der Textanalyse im Aufsatzunterricht der Unterprima fest zu verankern. In dem Beitrag soll gezeigt werden, unter welchen Bedingungen die Abhandlung in lateinischer Sprache durch den Reflexionsaufsatz in französischer Sprache abgelöst wurde. Auch geht es 
darum, zu hinterfragen, warum dieser Prozess das Abitur an sich in eine tiefe Krise stürzte, und mit welchen Mitteln diese aufgelöst wurde.

INDEX

Index géographique : France

Index chronologique : XIXe siècle, XIXe siècle (1870-1914)

Mots-clés : baccalauréat, composition française, composition latine, dissertation, examen, exercice scolaire, sujet d'examen

\section{AUTEUR}

ANDRÉ CHERVEL

Service d'histoire de l'éducation

INRP/CNRS 